\title{
THE HAUSDORFF DIMENSION OF A SET OF NORMAL NUMBERS II
}

\author{
A. D. POLLINGTON
}

(Received 14 October 1986)

Communicated by W. Moran

\begin{abstract}
Let $R, S$ be a partition of $2,3, \ldots$ so that rational powers fall in the same class. Let $\left(\lambda_{n}\right)$ be any real sequence; we show that there exists a set $N$, of dimension 1 , so that $\left(x+\lambda_{n}\right)$ $(n=1,2, \ldots)$ are normal to every base from $R$ and to no base from $S$, for every $x \in N$.

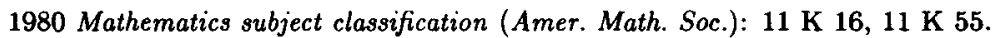

Keywords and phrases: normal numbers, Hausdorff dimension, sum sets.
\end{abstract}

\section{Introduction}

We call two natural numbers $r, s$ equivalent, $r \sim s$, when each is a rational power of the other. Schmidt [4] showed that given any partition of the numbers $2,3, \ldots$ into two disjoint classes $\mathbf{R}, \mathbf{S}$, so that equivalent numbers fall in the same class, then there is an uncountable set, $N$, of numbers which are normal to every base from $\mathbf{R}$ and to no base from S. In [3] we showed that the set of numbers with this property has Hausdorff dimension 1.

Pearce and Keane [2] gave a new proof of Schmidt's result. Given $r, s, r \nsim s$, there are uncountably many numbers which are normal to base $r$ but not even simply normal to base $s$. Brown, Moran and Pearce [1], have recently shown, using the theory of Riesz product measures, that every real number can be expressed as the sum of four numbers none of which is normal to base $s$ but all of which are normal to every base $r \nsim s$.

(C) 1988 Australian Mathematical Society $0263-6115 / 88 \$ A 2.00+0.00$ 
In this paper we return to the method used by Schmidt [4] and the author [3] and prove

THEOREM 1. Given any partition of the numbers $2,3, \ldots$ into two disjoint classes $\mathbf{R}, \mathbf{S}$ so that equivalent numbers fall into the same class, and any real sequence $\left(\lambda_{i}\right)_{i \in N}$, then the set of numbers $\xi$ for which $\lambda_{i}+\xi(i=1,2, \ldots)$ is normal to every base from $\mathbf{R}$ and to no base from $\mathbf{S}$ has Hausdorff dimension 1 .

This extends the results of [3] to simultaneous translates. It follows immediately from Theorem 1 that every real number can be expressed as a sum of two numbers from $N$.

\section{Preliminaries}

The proof of this result proceeds along the same lines as that given in [3]. The only changes that need to be made are in that part of the argument concerned with the non-normality with respect to the bases from $\mathbf{S}$, the construction of the sets $J_{1} \supset J_{2} \supset \cdots$. As before we apply our construction to bases $\geq A$, which gives us a Hausdorff dimension of $\log (A-3) / \log A$, taking unions over $A$ gives dimension 1. We assume that our sequences $\mathbf{R}=\left(r_{1}, r_{2}, \ldots\right), \mathbf{S}=\left\{s_{1}, s_{2}, \ldots\right\}$ satisfy the conditions of Section 3 of [3].

We write $h(m)$ for the least number $h$, for which

$$
m \neq \equiv 0\left(\bmod 2^{h}\right), \quad \text { that is, } m=2^{h} \cdot k+2^{h-1},
$$

and let

$$
g(m)=h(k)
$$

Put

$$
s(m)=s_{g(m)}, \quad \lambda(m)=\lambda_{h(m)} .
$$

Then as $m$ runs through the natural numbers, with the non-negative integer powers of 2 deleted, each $\lambda_{j}$ appears infinitely often in $\lambda(m)$, and as $m$ runs through those numbers for which $\lambda(m)=\lambda_{j}$ each $s_{i}$ appears infinitely often in the sequence $s(m)$.

\section{Construction of a set of nonnormal numbers}

We construct sets $J_{0}=[0,1] \supset J_{1} \supset J_{2} \supset \cdots$, each the union of closed intervals. Let $f(m)=e^{\sqrt{ } m}+2 s_{1} m^{3}$. Put

$$
\langle m\rangle=\lceil f(m)\rceil, \quad\langle m ; x\rangle\lceil\langle m\rangle / \log x\rceil
$$


where $\lceil x\rceil=-[-x]$.

$$
b_{m}=\langle m+1 ; s(m)\rangle
$$

and

$$
a_{m+1}=\left[\frac{b_{m} \log s(m)}{\log s(m+1)}\right]+2
$$

Then

$$
\frac{\langle m+1\rangle}{\log s(m+1)}+2 \leq a_{m+1} \leq \frac{\langle m+1\rangle}{\log s(m+1)}+\log \log m+3
$$

and

$$
s(1)^{b_{1}}<s(2)^{a_{2}}<s(2)^{b_{2}}<s(3)^{a_{3}}<\cdots .
$$

Let $J_{1}$ be the union of the intervals, each of length $s(1)^{-b_{1}}$, whose left end points are of the form

$$
\xi_{1}=\frac{\varepsilon_{1}}{s(1)}+\cdots+\frac{\varepsilon_{b_{1}}}{s(1)^{b_{1}}}-\lambda(1)
$$

where the $\varepsilon_{i}$ range over $0,1, \ldots, s(1)-\delta(1)$ and

$$
\delta(i)= \begin{cases}2 & \text { if } s(i) \text { is odd } \\ 3 & \text { if } s(i) \text { is even }\end{cases}
$$

There are $(s(1)-\delta(1))^{b_{1}}$ such intervals $I$ of $J_{1}$.

Suppose that $J_{k}$ has been constructed and that $I_{k}$ is an interval of $J_{k}$ of length $s(k)^{-b_{k}}$. By (5)

$$
s(k+1)^{-a_{k+1}+2} \leq s(k)^{-b_{k}} .
$$

Thus in each interval $I_{k}$ there are at least

$$
\left[\frac{s(k+1)^{a_{k+1}}}{s(k)^{b_{k}}}\right]-2
$$

intervals $I_{k}^{\prime}$ of length $s(k+1)^{-a_{k+1}}$ with left end point, $\rho_{k}$, for which $\rho_{k}+\lambda(k+1)$ is a finite decimal of length $a_{k+1}$ in base $s(k+1)$. We construct subintervals of $I_{k}^{\prime}$ of length $s(k+1)^{-b_{l k+1}}$ whose left end points are of the form

$$
\xi_{k+1}=\rho_{k}+\left(\frac{\varepsilon_{1}}{s(k+1)}+\cdots+\frac{\varepsilon_{t_{k+1}}}{s(k+1)^{t_{k+1}}}\right) s(k+1)^{-a_{k+1}}
$$

where $t_{k}=b_{k}-a_{k}$ and the $\varepsilon_{i}$ can range over $0,1, \ldots, s(k+1)-\delta(k+1)$.

In each interval $I_{k}^{\prime}$ there are $(s(k+1)-\delta(k+1)+1)^{t_{k+1}}$ such intervals. Let $J_{k+1}$ be the union of all such intervals taken over all $I_{k}^{\prime}$. Then $J_{k+1}$ is the union of at least

$$
\left(\left[\frac{s(k+1)^{a_{k+1}}}{s(k)^{b_{k}}}\right]-2\right)(s(k+1)-\delta(k+1)+1)^{t_{k+1}}
$$

intervals of length $s(k+1)^{-b_{k+1}}$. This completes the construction of the sequence of sets $J_{0} \supset J_{1} \supset \cdots$. 
LEMMA 1. If $\xi \in \bigcap_{i=1}^{\infty} J_{i}$ then $\xi+\lambda_{j}$ is non-normal to each base $s_{1}, s_{2}, \ldots$, for every $j \in N$.

ProOF. Fix $g, h$ and let $\lambda=\lambda_{h}, s=s_{g}$. Let $q$ be so large that

$$
\left(\frac{s-1}{s}\right)^{q}<2^{-g-h}
$$

For a number $M$ with $h(M)=h, g(M)=g$ there are at least,

$$
\sum_{\substack{m \leq M \\ h(m)=h \\ g(m)=g}}\left(t_{m}-1-q\right),
$$

$q$-blocks $\varepsilon_{i+1} \cdots \varepsilon_{i+q}$ consisting of the digits $0,1, \ldots, s-2$ in the expansion of $\xi+\lambda$, for which $i+q \leq b_{M}$. Now $h(m)=h$ if $m=2^{h} \cdot k+2^{h-1}$ and $g(m)=g$ if $k=2^{g} \cdot l+2^{g-1}$ so $m=2^{h+g} l+2^{h+g-1}+2^{h-1}$, that is,

$$
m \equiv 2^{h+q-1}+2^{h-1} \quad\left(\bmod 2^{g+h}\right) .
$$

$$
\begin{aligned}
& \text { If } g(m)=g, h(m)=h \text { and } m>2^{h+g-1}+2^{h-1} \text {, then, by (6) } \\
& t_{m}-1-q \geq 2^{-g-h} \sum_{j=m-2^{g+h}+1}^{m}[(\langle j+1 ; \delta\rangle-\langle j ; s\rangle)-\log \log m-5-q]
\end{aligned}
$$

since $t_{m}=b_{m}-a_{m}$ and $\langle m+1 ; s\rangle-\langle m ; s\rangle$ is a non-decreasing function of $m$. Thus (10) is at least

$$
\begin{aligned}
& \sum_{\substack{m \leq M \\
g(m)=g \\
h(m)=h}} \sum_{j=m-2^{g+h}+1}^{m}((\langle j+1 ; s\rangle-\langle j ; s\rangle)-\log \log m-5-q) \\
& \geq 2^{-g-h}((M+1 ; s\rangle-\langle 1 ; s\rangle-M(\log \log M+5-q)) \\
& \quad=2^{-g-h} b_{M}(1+O(1)) .
\end{aligned}
$$

If $\xi+\lambda$ were normal to base $s$, the number of $q$-blocks with digits $0,1, \ldots, s_{q}-2$ and indices smaller than $b_{M}$ would be asymptotic to $((s-1) / s) b_{M}$. By $(9)$ this is clearly not the case and Lemma 1 is proved.

\section{Construction of a set of normal numbers}

We also have to ensure that the translate of the numbers we have constructed are also all normal to every base from $\mathbf{R}$. We do this, as in [3], by discarding certain of the intervals $J_{i}$ at each stage, to obtain a new sequence, $K_{1} \supset K_{2} \supset$ $\cdots$, with $K_{i} \supset J_{i}$. 
Consider the intervals $I_{m-1}^{\prime}$. In each such interval there are

$$
(s(m)-\delta(m)+1)^{t_{m}}
$$

intervals of $J_{m}$ whose left end points we denote by $\xi_{m}$. Let

$$
A_{m}(x)=\sum_{t=-m}^{m} \sum_{i=1}^{m}\left|\sum_{j=\left\langle m_{j} r_{i}\right\rangle+1}^{\left\langle m+1 ; r_{i}\right\rangle} e\left(r_{i}^{j} t x\right)\right|^{2} .
$$

LEMMA 2. Let $j \in N$, then if $m \geq \delta_{j}$ there are at least $(s(m)-3)^{t_{m}}$ numbers $\xi_{m} \in I_{m-1}^{\prime}$ for which

$$
A_{m}\left(\xi_{m}+\lambda_{i}^{2}\right) \leq c m(\langle m+1\rangle-\langle m\rangle)^{2-\beta_{m} / 2},
$$

for $i=1, \ldots, j$. Here $c$ is an absolute constant and $\delta_{j}$ is constant depending on $j$. Here $\beta_{m}$ is as in [3], $\beta_{m} \geq \beta_{1} m^{-1 / 4}$.

ProOF. Let $s=s(m)$. As in the proof of Lemma 3 of [3] we have:

The number of $\xi_{m} \in I_{m-1}^{\prime}$ for which

$$
A_{m}\left(\xi_{m}+\lambda_{j}\right)>c m^{2}(\langle m+1\rangle-\langle m\rangle)^{2-\beta_{m} / 2}
$$

is at most

$$
(\langle m+1\rangle-\langle m\rangle)^{-\beta_{m} / 2}(s-\delta+1)^{t_{m}} .
$$

But $\beta_{m} \geq \beta_{1} m^{-1 / 4}$ and $(\langle m+1\rangle-\langle m\rangle) \geq e^{\sqrt{ } m} /(2 \sqrt{ } m+1)$, and so

$$
(\langle m+1\rangle-\langle m\rangle)^{-\beta_{m} / 2}<\frac{1}{2^{j+1}} \text { for } m>\delta_{j} .
$$

Hence there are least $(s-\delta+1)^{t_{m}} / 2$ numbers $\xi_{m} \in I_{m-1}^{\prime}$ for which

$$
A_{m}\left(\xi_{m}+\lambda_{i}\right) \leq c m^{2}(\langle m+1\rangle-\langle m\rangle)^{2-\beta_{m} / 2}, \quad i=1,2, \ldots, j .
$$

For $m \geq \delta_{j}(s-3)^{t_{m}}<(s-\delta+1)^{t_{m}} / 2$, this proves the lemma.

We construct the sequence of sets $K_{1} \supset K_{2} \supset \cdots$ in the same way as $J_{1} \supset J_{2} \supset \cdots$ was constructed. But at each stage in our construction of $\left\{K_{m}\right\}$ we use only the $(s(m)-3)^{t_{m}}$ points $\xi_{m}$ satisfying Lemma 2. The remainder of the proof of Theorem 1 now proceeds exactly as in [3].

By a straightforward application of Weyl's criterion we have

COROLlARY 1. Given $\left(a_{i}\right)$ a sequence of non-zero rational numbers, $\left(b_{i}\right)$ a sequence of real numbers and a partition $\mathbf{R}, \mathbf{S}$ of the numbers $2,3, \ldots$ so that equivalent numbers fall into the same class, then the set of numbers $\xi$ for which $a_{i} \xi+b_{i}$ is normal to every base from $\mathbf{R}$ and to no base from $\mathbf{S}$ has Hausdorff dimension 1.

COROllary 2. Given any partition of $2,3, \ldots$ into two classes $\mathbf{R}, \mathbf{S}$, so that equivalent numbers fall into the same class, then every real number can be 
written as a sum of two numbers both normal to every base from $\mathbf{R}$ and to no base from $\mathbf{S}$.

\section{Bibliography}

[1] G. Brown, W. Moran, and C. E. M. Pearce, 'Riesz products and normal numbers', J. London Math. Soc. (2) 32 (1985), 12-18.

[2] C. E. M. Pearce and M. S. Keane, 'On normal numbers', J. Austral. Math. Soc. (1) 32 (1982), 79-87.

[3] A. D. Pollington, 'The Hausdorff dimension of a set of normal numbers', Pacific J. Math. 95 (1980) 193-204.

[4] W. M. Schmidt, 'Über die Normalität von Zahlen zu verschiedenen Basen', Acta Arith. 7 (1961-62), 299-309.

\section{Department of Mathematics \\ Brigham Young University \\ Provo, Utah 84602 \\ U.S.A.}

